\title{
静叶调节机构尺度全局优化设计方法研究*
}

\author{
唐佑远 郭为忠 \\ (上海交通大学机械系统与振动国家重点实验室 上海 200240)
}

\begin{abstract}
摘要: 为避免航空发动机喘振, 高压压气机的可调静叶需要按照拟定的调节规律转动。静叶调节机构是驱动可调静叶转动的 执行机构, 一般采用多级联动调节的方式。由于拟定的静叶调节规律十分复杂, 且多级静叶调节机构之间运动学耦合, 静叶 调节机构的尺度设计问题非常困难。目前一般采取逐级分解、单级优化的局部优化策略设计机构参数, 无法获得全局最优的 机构设计参数。为提高静叶调节精度, 通过分析静叶调节机构运动学特点, 提出正-逆运动学结合的机构尺度全局优化设计方 法: 先进行基于逆运动学解析解的全局、高效参数优化设计, 再进行基于正运动学数值解的机构参数高精度优化求解, 有效 解决了静叶调节机构的全局尺度优化设计难题。通过案例分析, 验证了所提设计方法的有效性和实用性。
\end{abstract}

关键词: 高压压气机; 静叶调节机构; 机构尺度优化; 正-逆运动学结合; 全局优化

中图分类号: TG156

\section{Global Dimensional Optimization for the Design of Adjusting Mechanism of Variable Stator Vanes}

\section{TANG Youyuan GUO Weizhong}

(State Key Laboratory of Mechanical System and Vibration, Shanghai Jiao Tong University, Shanghai 200240)

\begin{abstract}
In order to avoid the problem of compressor surge, the variable stator vane (VSV) needs to follow the rules established by hydromechanics. The VSV is normally composed of several connected stages and actuated by one adjusting mechanism that contains several coupled spatial linkage mechanism units. It is difficult to perform the dimensional synthesis of the VSV adjusting mechanism since this mechanism is a coupled system of the several spatial linkage units and the rules established by hydromechanics are also complicated. Currently, a divide and conquer method is applied to synthesize the dimensions of the VSV adjusting mechanism by synthesizing the spatial linkage mechanism units one by one, hereafter the global optimization is unable to be achieved. In order to improve the VSV's adjustment accuracy, a novel global dimensional synthesis method is proposed by combining the forward kinematics (FK) and inverse kinematics (IK) considering the analytical solvability of the FK and IK models. An efficiently global optimization is achieved firstly to provide the initial solution by using the analytical IK model and then a global optimization of high accuracy is achieved by searching from the initial solution based on the numerical FK model. A case study is given to demonstrate the proposed method.
\end{abstract}

Key words: high pressure compressor; adjusting mechanism of variable stator vanes; dimensional optimization; combined forward and inverse kinematics; global optimization

\section{0 前言}

高压压气机是航空发动机的核心部件之一，高 压压气机维持稳定、可靠的工作状态对发动机可靠 运行至关重要。当高压压气机工作在非设计点时, 容易发生发动机喘振, 甚至导致发动机故障, 因此 高压压气机一般采用静叶可调技术 ${ }^{1]}$, 根据发动机

* 国家自然科学基金(51735009)资助项目。20191011 收到初稿, 20200214 收到修改稿
工况实时调节静叶的角度，有效避免喘振发生。

静叶调节机构一般采用多级联动调节方式 ${ }^{[2]}$,

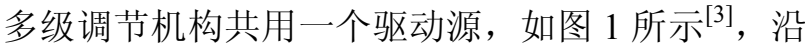
着气流方向按顺序定义静叶调节机构的级别, 每一 级静叶调节机构构型相同, 图中以第 $j$ 级静叶调节 机构为例, 分析了单级静叶调节机构的组成。静叶 调节机构采用多级联动调节的形式, 降低了驱动的 数量, 提高了压气机的工作可靠性; 但多级联动调 节也使得多级调节机构之间运动耦合、数学模型更 加复杂, 导致机构尺度全局优化设计时计算量巨大、 优化设计效率很低 ${ }^{[4]}$ 。 


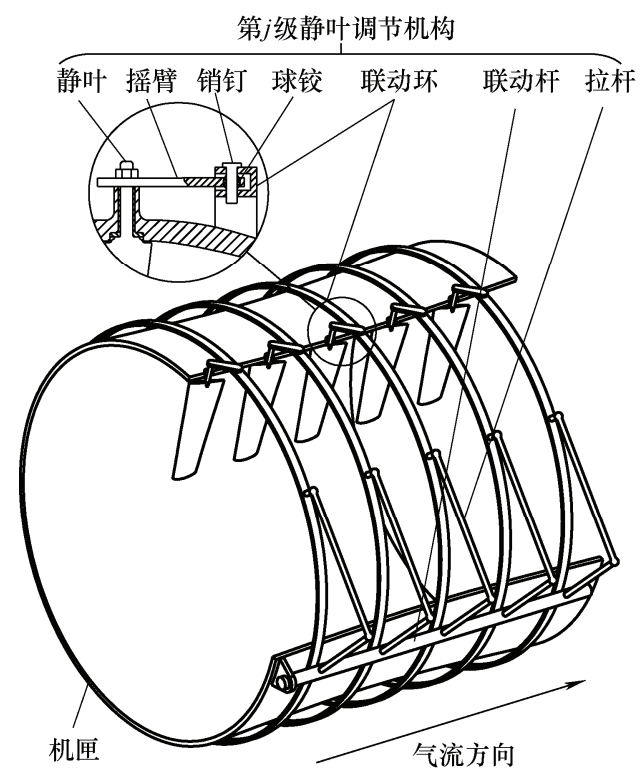

图 1 静叶调节机构

静叶调节机构的尺度优化设计问题十分复杂。 针对多级联动调节特点, 通常采取将静叶调节机构 逐级分解、单级进行尺度局部优化的设计策略 ${ }^{[4-7]}$, 将多级联动调节的静叶调节机构设计过程拆分, 每 次只设计某一级静叶调节机构参数。这种局部优化 设计策略降低了设计参数的规模, 但无法实现全局 优化设计, 导致静叶调节精度低, 严重影响高压压 气机的压比、可靠性等性能指标 ${ }^{[8]}$ 。

目前, 静叶调节机构的尺度优化设计方法可以 分为两类, 一类是采用 ADAMS、ISIGHT 等工程软 件进行参数设计 ${ }^{[4-6]}$, 计算效率和设计精度均较低, 一般设计误差为 $0.6^{\circ} \sim 0.7^{\circ}$; 另一类是构建数学模 型进行优化的方法, 如文献[7]推导了静叶调节机构 的运动学方程, 采用数学模型对静叶调节机构进行 优化设计, 设计误差最好时能控制在 $0.4^{\circ}$ 左右。

综上所述, 目前的静叶调节机构采取将多级机 构逐级分解、每次只进行单级优化的局部设计策略, 通过工程软件或数学建模的方法只能对单级机构尺 度进行优化, 无法实现静叶调节机构的尺度全局优 化设计, 整体设计误差偏大。

为从根本上解决静叶调节机构尺度全局优化设 计难题, 本文在分析静叶调节机构运动学模型特点 的基础上, 提出基于机构正-逆运动学相结合进行尺 度设计的全局优化设计方法, 将静叶调节机构尺度 优化设计过程分解为先进行基于逆运动学解析解的 高效尺度全局优化求解、再进行基于正运动学数值 解的高精度尺度全局优化求解这两个相互衔接的计 算过程, 有效解决了静叶调节机构尺度全局优化设 计存在的计算量巨大、优化设计效率低的问题。通 过某型静叶调节机构的尺度优化设计案例, 实现了
35 个设计目标点的误差均控制在 $\pm 0.15^{\circ}$ 以内的目 标，验证了所提设计方法的有效性和实用性。

\section{1 静叶调节机构的设计目标分析}

静叶调节装置的设计过程比较复杂, 一般分成 两步(图 2), 步骤(1)利用燃烧力学和气动力学原理确 定静叶叶形和调节规律, 步骤(2)进行机构尺度参数 和驱动规律设计。步骤(2)中尺度优化设计的任务是 通过设计机构尺度参数值, 使静叶在静叶调节机构 驱动下, 能按照拟定的静叶调节规律转动。步骤(1) 中确定的静叶调节规律即为步骤(2)中静叶调节机 构尺度优化设计需要实现的设计目标。
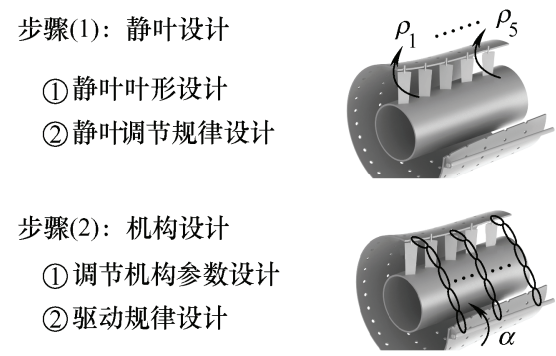

图 2 静叶调节装置的设计步骤

\section{1 静叶调节机构的尺度优化设计目标}

静叶调节机构尺度优化的设计目标是要实现静 叶调节规律。静叶调节规律的确定比较复杂, 一般 以燃烧力学和气动力学为理论基础 ${ }^{[9-10]}$, 构建静叶 一维压气机特性目标函数 ${ }^{[11]}$, 通过分析压气机整体 的喘振边界，确定最佳调节规律 ${ }^{[12-13]}$ 。文献[14]在 讨论静叶调节规律对高压压气机性能影响时, 给出 一组非常复杂的静叶调节规律, 该调节规律有 5 级, 每一级静叶调节规律用 7 个离散设计点表示, 如表 1 所示 ${ }^{[14]}$ 。

\section{表 1 静叶调节规律}

\begin{tabular}{ccccccc}
\hline \multirow{2}{*}{$\begin{array}{c}\text { 驱动位置 } \\
\text { 序号 } i\end{array}$} & 驱动规律 & \multicolumn{5}{c}{ 静叶调节规律 $\rho$} \\
\cline { 3 - 7 } & $\alpha$ & $\rho_{1}$ & $\rho_{2}$ & $\rho_{3}$ & $\rho_{4}$ & $\rho_{5}$ \\
\hline 1 & $\alpha_{1}$ & 0 & 0 & 0 & 0 & 0 \\
2 & $\alpha_{2}$ & 7.74 & 6 & 6.15 & 3.8 & 3.95 \\
3 & $\alpha_{3}$ & 16.77 & 13 & 13.32 & 8.32 & 8.65 \\
4 & $\alpha_{4}$ & 25.69 & 20 & 20.48 & 12.9 & 13.41 \\
5 & $\alpha_{5}$ & 42.21 & 33 & 32.55 & 21.42 & 22.25 \\
6 & $\alpha_{6}$ & 51.24 & 40 & 39.55 & 25.94 & 26.95 \\
7 & $\alpha_{7}$ & 64.62 & 50 & 49.55 & 32.22 & 33.48 \\
\hline
\end{tabular}

表 1 中 $\alpha$ 表示静叶调节机构的驱动规律, $\rho$ 表 示 5 级静叶调节规律, $\rho_{1} 、 \rho_{2} 、 \rho_{3} 、 \rho_{4} 、 \rho_{5}$ 分别 表示第 $1 \sim 5$ 级静叶调节规律。为不失一般性, 定义 静叶调节规律符号表示如下 


$$
\boldsymbol{\rho}=\left[\begin{array}{ccccc}
\rho_{1,1} & \cdots & \rho_{1, j} & \cdots & \rho_{1, n} \\
\vdots & & \vdots & & \vdots \\
\rho_{i, 1} & \cdots & \rho_{i, j} & \cdots & \rho_{i, n} \\
\vdots & & \vdots & & \vdots \\
\rho_{m, 1} & \cdots & \rho_{m, j} & \cdots & \rho_{m, n}
\end{array}\right]
$$

式中, $\rho_{i, j}$ 表示第 $j$ 级、第 $i$ 个驱动位置的静叶角度 值, $i$ 表示单级静叶调节规律设计目标的位置序号, 取值范围是 $i=1,2, \cdots, m ; j$ 表示静叶调节规律所在 的级别, 取值范围是 $j=1,2, \cdots, n$ 。

多级联动调节静叶调节机构的驱动规律符号表 示如下

$$
\boldsymbol{\alpha}=\left[\begin{array}{lllll}
\alpha_{1} & \cdots & \alpha_{i} & \cdots & \alpha_{m}
\end{array}\right]^{\mathrm{T}}
$$

式中， $\alpha_{i}$ 表示第 $i$ 个驱动位置的联动杆的角位移; $i$ 表示驱动的位置序号, 取值范围是 $i=1,2, \cdots, m$ 。

\section{2 静叶调节机构尺度优化设计目标的特点}

以表 1 中静叶调节规律为例, 需要设计 5 级联 动的静叶调节机构尺度参数和驱动参数, 使得驱动 连杆转动到第 $i$ 个驱动位置 $\alpha_{i}$ 时, 对应的第 $1 \sim 5$ 级 静叶角度分别为 $\rho_{i, 1} 、 \rho_{i, 2} 、 \rho_{i, 3} 、 \rho_{i, 4} 、 \rho_{i, 5}$ 。

由于静叶调节规律具有级间变化非线性、设计 目标点多、多级设计目标耦合等特殊性, 导致设计 一套静叶调节机构来同时满足表 1 中的 35 个设计目 标点, 是一个十分复杂且困难的任务。

\section{2 联调式静叶调节机构运动学建模}

多级联调式静叶调节机构运动学建模分为单级 静叶调节机构运动学模型构建和多级运动学模型构 建两个步骤, 首先, 建立了单级静叶调节机构的运 动学模型。单级静叶调节机构的运动学模型又分为 RSSCCSR 机构的运动学构建和单级静叶攻角运动 学模型的构建。其次, 通过将多级静叶调节机构运 动学驱动耦合, 构造多级联调式静叶调节机构的运 动学模型。

\section{1 静叶调节机构构型与自由度分析}

\subsection{1 静叶调节机构模型构建}

分析图 1 所示静叶调节机构, 可以绘制图 3 所 示的机构运动简图, 其中以第 $j$ 级静叶调节机构为 例, 分析机构模型建立的过程。联动杆 $A_{1} A_{n}$ 是静叶 调节机构的主动件, 可以绕着轴线旋转; 拉杆 $B_{j} C_{j}$ 一端通过 $\mathrm{S}$ 副与联动杆 $A_{1} A_{n}$ 上固连的连杆 $A_{j} B_{j}$ 连 接, 另一端通过 $\mathrm{S}$ 副与联动环的 $C_{j}$ 点相连, 拉杆 $B_{j} C_{j}$ 的主要作用是将联动杆 $A_{1} A_{n}$ 的旋转运动传递 给每一级联动环, 使得联动环沿着发动机轴线转动 和移动; 联动环与摇臂 $F_{j} G_{j}$ 通过带球铰的销钉 $E_{j} F_{j}$
相连，销钉 $E_{j} F_{j}$ 与联动环之间以 $\mathrm{C}$ 副连接, 销钉 $E_{j} F_{j}$ 与摇臂之间以 $\mathrm{S}$ 副连接; 摇臂 $F_{j} G_{j}$ 与静叶轴 颈固定连接, 静叶可以绕着叶片轴线旋转。

图 3 中单级静叶调节机构是 RSSCCSR 型六杆 机构。由于静叶调节机构采用多级联动调节的形式, 多级静叶调节机构共用一个旋转副, 故 5 级静叶调 节机构构型是 R-5(SSCCSR)。

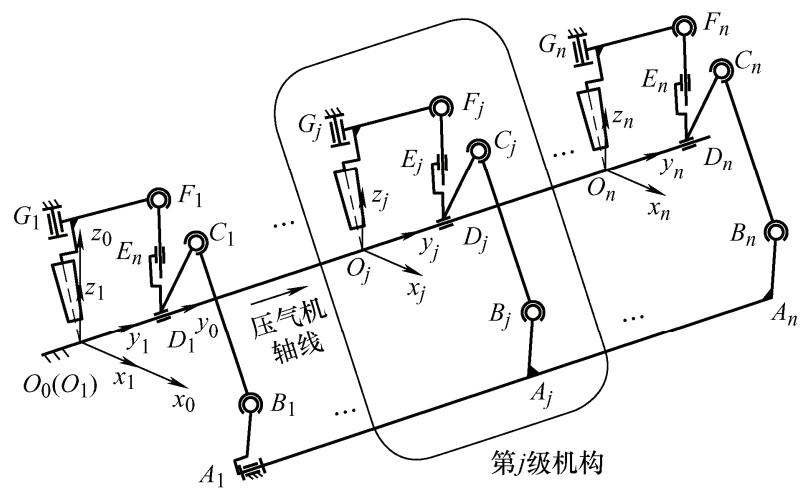

图 3 多级联动调节的静叶调节机构简图

\subsection{2 静叶调节机构自由度分析}

R- $n$ (SSCCSR)机构构件数量 $N=4 n+2$, 运动副 数量 $g=6 n+1$, 所有运动副的自由度之和 $\sum_{i=1}^{g} f_{i}=$ $14 n+1$, 机构过约束数 $\mu=0$ ，局部自由度 $\zeta=2 n$, 根据自由度计算公式, 可以求得 R- $n(\mathrm{SSCCSR})$ 机构 自由度是 1 , 计算公式如下

$$
\begin{aligned}
& M=6(N-g-1)+\sum_{i=1}^{g} f_{i}+\mu-\zeta= \\
& 6 \times(4 n+2-6 n-1-1)+14 n+0-2 n=1
\end{aligned}
$$

静叶调节机构由多级构型相同的机构组成, 且 机构自由度为 1 。为便于单级静叶调节机构运动学 建模, 将单级静叶调节机构分为 RSSCCSR 机构运 动学模型和静叶安装参数两部分, 下面以第 $j$ 级静 叶调节机构为例分析静叶调节机构的运动学模型。

\section{2 单级 RSSCCSR 机构运动学建模}

2.2.1 单级 RSSCCSR 机构坐标系建立与参数定义 如图 4 所示建立第 $j$ 级 RSSCCSR 机构、在第 $i$

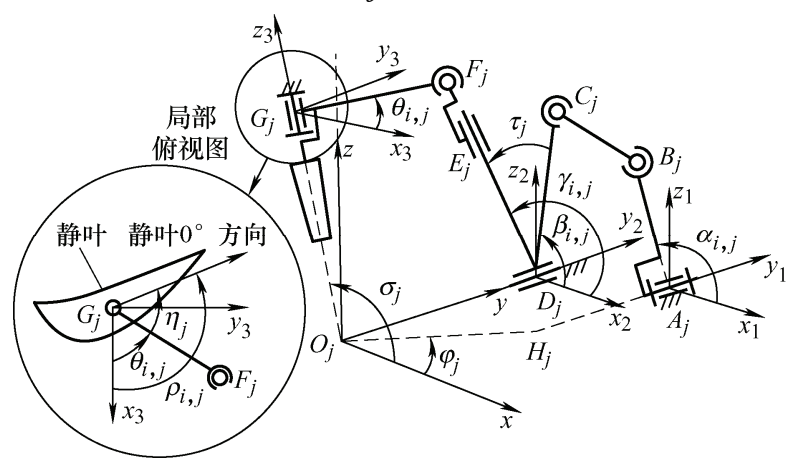

图 4 第 $j$ 级调节机构 RSSCCSR 参数的定义 
个驱动位置时的全局坐标系和局部坐标系, 参数定 义和静叶安装参数关系示于表 2 和表 3 。

\section{表 2 第 $j$ 级 RSSCCSR 机构坐标系定义}

\begin{tabular}{|c|c|}
\hline 坐标系 & 定义规则 \\
\hline & 多级联动调节静叶调节机构的基坐标系, 坐标系原点定 \\
\hline$O_{0} x_{0} y_{0} z_{0}$ & 义在第 1 级静叶轴线与压气机轴线的交点 $O_{0}$ 上, $x_{0}$ 轴沿 \\
\hline & $\begin{array}{l}\text { 着压气机水平方向, } y_{0} \text { 轴沿着压气机轴线方向 } \\
\text { 单级调节机构的全局坐标系, 坐标系原点定义在本级静 }\end{array}$ \\
\hline$O_{j} x y z$ & $\begin{array}{l}\text { 叶轴线与压气机轴线的交点 } O_{j} \text { 上, } x \text { 轴沿着压气机水平 } \\
\text { 方向, } y \text { 轴沿着压气机轴线方向 }\end{array}$ \\
\hline$A_{j} x_{1} y_{1} z_{1}$ & $\begin{array}{l}\text { 局部坐标系, 坐标系原点在 } A_{j} \text { 上, 坐标轴的方向与全 } \\
\text { 局坐标系 } O_{j} x y z \text { 相同 }\end{array}$ \\
\hline$D_{j} x_{2} y_{2} z_{2}$ & $\begin{array}{l}\text { 局部坐标系, 坐标系原点在 } D_{j} \text { 上, 坐标轴的方向与全 } \\
\text { 局坐标系 } O_{j} x y z \text { 相同 }\end{array}$ \\
\hline$G_{j} x_{3} y_{3} z_{3}$ & $\begin{array}{l}\text { 局部坐标系, 坐标系原点在 } G_{j} \text { 上, 坐标轴的方向与全 } \\
\text { 局坐标系 } O_{j} x y z \text { 相同 }\end{array}$ \\
\hline
\end{tabular}

\section{表 3 第 $\boldsymbol{j}$ 级 RSSCCSR 机构参数定义}

\begin{tabular}{|c|c|c|}
\hline 序号 & $\begin{array}{c}\text { 机构参数 } \\
\text { 符号 }\end{array}$ & 定义规则 \\
\hline & & 静叶调节机构第 $i$ 个驱动位置的输入角, 即连 \\
\hline 1 & $\alpha_{i, j}$ & $\begin{array}{l}\text { 杆 } A_{j} B_{j} \text { 与 } x_{1} \text { 轴的夹角, 沿着 } x_{1} \text { 轴正方向是 } 0^{\circ}, \\
\text { 绕着箭头方向是正方向 }\end{array}$ \\
\hline 2 & $\varphi_{j}$ & $\begin{array}{l}\text { 直线 } H_{j} O_{j} \text { 与 } x \text { 轴的夹角, 沿着 } x \text { 轴正方向 } \\
\text { 是 } 0^{\circ} \text {, 绕着箭头方向是正方向 }\end{array}$ \\
\hline 3 & $\beta_{i, j}$ & $\begin{array}{l}\text { 在第 } i \text { 个驱动位置, 连杆 } C_{j} D_{j} \text { 与 } x_{2} \text { 轴的夹 } \\
\text { 角, 沿着 } x_{2} \text { 轴正方向是 } 0^{\circ} \text {, 绕着箭头方向是正 } \\
\text { 方向 }\end{array}$ \\
\hline 4 & $\gamma_{i, j}$ & $\begin{array}{l}\text { 在第 } i \text { 个驱动位置, 连杆 } D_{j} E_{j} \text { 与 } x_{2} \text { 轴的夹 } \\
\text { 角, 沿着 } x_{2} \text { 轴正方向是 } 0^{\circ} \text {, 绕着箭头方向是正 } \\
\text { 方向 }\end{array}$ \\
\hline 5 & $\tau_{j}$ & $\begin{array}{l}\text { 连杆 } C_{j} D_{j} \text { 与 } D_{j} E_{j} \text { 的夹角, 绕着箭头方向是 } \\
\text { 正方向 }\end{array}$ \\
\hline 6 & $\sigma_{j}$ & $\begin{array}{l}\text { 轴线 } G_{j} O_{j} \text { 与 } x \text { 轴的夹角, 沿着 } x \text { 轴正方向 } \\
\text { 是 } 0^{\circ} \text {, 绕着箭头方向是正方向 }\end{array}$ \\
\hline 7 & $\theta_{i, j}$ & $\begin{array}{l}\text { 在第 } i \text { 个驱动位置, 连杆 } F_{j} G_{j} \text { 与 } x_{3} \text { 轴的夹 } \\
\text { 角, 沿着 } x_{3} \text { 轴正方向是 } 0^{\circ} \text {, 绕着箭头方向是正 } \\
\text { 方向 }\end{array}$ \\
\hline 8 & $\eta_{j}$ & $\begin{array}{l}\text { 连杆 } F_{j} G_{j} \text { 与静叶上 } 0^{\circ} \text { 方向线之间的夹角 (二 } \\
\text { 者固结为一体), 正号表示沿着箭头方向转动 } \\
\eta_{j} \text {, 负号表示绕着箭头所示反方向转动 } \eta_{j}, \\
\text { 且有 } \rho=\theta+\eta\end{array}$ \\
\hline 9 & $\rho_{i, j}$ & $\begin{array}{l}\text { 在第 } i \text { 个驱动位置、第 } j \text { 级静叶 } 0^{\circ} \text { 方向与 } x_{3} \text { 轴 } \\
\text { 的夹角, 沿着 } x_{3} \text { 轴正方向是 } 0^{\circ} \text {, 绕着箭头方向 } \\
\text { 是正方向 }\end{array}$ \\
\hline 10 & $l_{H_{j} O_{j}}$ & $A_{j}$ 点旋转副轴线与压气机轴线的距离 \\
\hline 11 & $l_{A_{j} H_{j}}$ & $\begin{array}{l}A_{j} \text { 点旋转副与 } O_{j} x z \text { 平面向量, 正号表示沿 } \\
\text { 着 } y_{0} \text { 轴的正方向, 负号表示沿着 } y_{0} \text { 轴的负 } \\
\text { 方向 }\end{array}$ \\
\hline 12 & $l_{A_{\beta} B_{j}}$ & 连杆 $A_{j} B_{j}$ 的长度 \\
\hline 13 & $l_{B, C_{j}}$ & 连杆 $B_{j} C_{j}$ 的长度 \\
\hline 14 & $l_{C_{j} D_{j}}$ & 连杆 $C_{j} D_{j}$ 的长度 \\
\hline 15 & $l_{D_{i} F_{i}}$ & $F_{j}$ 点球副与 $D_{j}$ 点圆柱副轴线的距离 \\
\hline
\end{tabular}

(续)

\begin{tabular}{ccc}
\hline 序号 & $\begin{array}{c}\text { 机构参数 } \\
\text { 符号 }\end{array}$ & \multicolumn{1}{c}{ 定义规则 } \\
\hline 16 & $l_{F_{F} G_{j}}$ & 连杆 $F_{j} G_{j}$ 的长度 \\
17 & $l_{G_{j} O_{j}}$ & $G_{j}$ 点旋转副与压气机轴线的距离 \\
18 & $l_{D O}$ & $D_{j}$ 点圆柱副与 $O_{j} x z$ 平面的距离 \\
\hline
\end{tabular}

\subsection{2 单级 RSSCCSR 机构运动学建模}

在静叶调节机构中, 从驱动到负载的运动学方 程定义为静叶调节机构的正运动学, 反之定义为逆 运动学, 故 RSSCCSR 机构的正运动学方程可以用 为 $\theta_{i, j}=g\left(\boldsymbol{x}_{j}, \alpha_{i, j}\right)$ 表示, 逆运动学方程可以用 $\alpha_{i, j}=h\left(\boldsymbol{x}_{j}, \theta_{i, j}\right)$ 表示, 式中 $\boldsymbol{x}_{j}$ 表示第 $j$ 级 RSSCCSR 机构参数构成的矢量。下面分别建立静叶调节机构 的正、逆运动学模型。

(1) RSSCCSR 机构正运动学建模。

对于静叶调节机构的正运动学, 根据定长条件 可以构造机构运动学方程组如下

$$
\left\{\begin{array}{c}
\left(X-\cos \beta_{i, j}\right)^{2}+\left(Y-\frac{l_{F_{j} G_{j}}}{l_{C_{j} D_{j}}} \sin \theta_{i, j}\right)^{2}+\left(Z-\sin \beta_{i, j}\right)^{2}=\frac{l_{B_{j} C_{j}}^{2}}{l_{C_{j} D_{j}}^{2}} \\
\cos \theta_{i, j}=-\frac{l_{G_{j} O_{j}}}{l_{F_{j} G_{j}}} \tan \left(\beta_{i, j}-\sigma_{j}+\tau_{j}\right) \\
X=\frac{1}{l_{C_{j} D_{j}}}\left(l_{H_{j} O_{j}} \cos \varphi_{j}+l_{A_{j} B_{j}} \cos \alpha_{i, j}\right) \\
Y=\frac{l_{A_{j} H_{j}}}{l_{C_{j} D_{j}}} \\
Z=\frac{1}{l_{C_{j} D_{j}}}\left(l_{H_{j} O_{j}} \sin \varphi_{j}+l_{A_{j} B_{j}} \sin \alpha_{i, j}\right)
\end{array}\right.
$$

根据式(4), 无法求出以 $\alpha_{i, j}$ 为自变量、 $\theta_{i, j}$ 为因 变量的正运动学 $\theta_{i, j}=g\left(\boldsymbol{x}_{j}, \alpha_{i, j}\right)$ 的解析解; 只能根 据上述方程组式(4)的关系式, 采用牛顿迭代法等数 值方法求解出正运动学的数值解。

(2) RSSCCSR 机构逆运动学建模。

文献[9]给出静叶调节机构运动学方程的详细 推导过程, 此处不再赘述。RSSCCSR 机构的逆运动 学解析式如下

$$
\begin{gathered}
\alpha_{i, j}=h\left(\boldsymbol{x}_{j}, \theta_{i, j}\right)=\arctan \left(\frac{\mp \frac{K_{1}}{K_{3}} \sqrt{U}+V}{ \pm \sqrt{U}+W}\right) \\
U=-\left(\left(U_{1}+U_{2}\right) l_{A_{j} B_{j}}^{4}+U_{3}\right) K_{3}^{2} \\
U_{1}=K_{3}^{4}+\left(2 K_{1}^{2}+2 K_{2}^{2}-2\right) K_{3}^{2} \\
U_{2}=K_{1}^{4}+\left(2 K_{2}^{2}-2\right) K_{1}^{2}+\left(K_{2}^{2}+1\right)^{2} \\
U_{3}=-2\left(K_{1}^{2}+K_{2}^{2}+K_{3}^{2}+1\right) l_{A_{j} B_{j}}^{2} l_{B_{j} C_{j}}^{2}+l_{B_{j} C_{j}}^{4}
\end{gathered}
$$




$$
\begin{gathered}
V=-K_{3}\left(K_{3}^{2} l_{A_{j} B_{j}}^{2}+\left(K_{1}^{2}+K_{2}^{2}+1\right) l_{A_{j} B_{j}}^{2}-l_{B_{j} C_{j}}^{2}\right) \\
W=-K_{1}^{3} l_{A_{j} B_{j}}^{2}+\left(\left(-K_{2}^{2}-K_{3}^{2}-1\right) l_{A_{j} B_{j}}^{2}+l_{B_{j} C_{j}}^{2}\right) K_{1} \\
K_{1}=\frac{l_{H_{j} O_{j}} \cos \varphi_{j}-l_{C_{j} D_{j}} \cos \beta_{i, j}}{l_{A_{j} B_{j}}} \\
K_{2}=\frac{l_{A_{j} H_{j}}-l_{D_{j} O_{j}}}{l_{A_{j} B_{j}}} \\
K_{3}=\frac{l_{H_{j} O_{j}} \sin \varphi_{j}-l_{C_{j} D_{j}} \sin \beta_{i, j}}{l_{A_{j} B_{j}}} \\
\beta_{i, j}=\gamma_{i, j}-\tau_{j} \\
\gamma_{i, j}=\arctan \left(\frac{-l_{F_{j} G_{j}} \cos \theta_{i, j}}{l_{G_{j} O_{j}}}\right)+\sigma_{j} \\
l_{D_{j} O_{j}}=l_{F_{j} G_{j}} \sin \theta_{i, j}
\end{gathered}
$$

2.2.3 第 $j$ 级静叶调节机构静叶攻角运动学模型

可调静叶在通过气动力学设计时会规定叶片上 的 $0^{\circ}$ 方向线, 通过分析图 4 中静叶 $0^{\circ}$ 方向矢量与 RSSCCSR 机构的输出 $F G$ 连杆的关系, 可知静叶上 $0^{\circ}$ 方向线相对于 $x_{3}$ 轴的夹角 $\rho_{i, j}$ 与机构角度 $\theta_{i, j}$ 存 在如下关系

$$
\rho_{i, j}=\theta_{i, j}+\eta_{j}
$$

根据静叶安装角的关系可以推导出第 $j$ 级静叶 攻角运动学模型。

(1) 第 $j$ 级静叶调节机构正运动学模型。

据式(4)、(6)可得静叶调节机构的正运动学模型

$$
\rho_{i, j}=g\left(\boldsymbol{x}_{j}, \alpha_{i, j}\right)+\eta_{j}
$$

(2) 第 $j$ 级静叶调节机构逆运动学模型。

据式(5)、(6)可得静叶调节机构的逆运动学模型

$$
\alpha_{i, j}=h\left(\boldsymbol{x}_{j}, \rho_{i, j}-\eta_{j}\right)
$$

\section{3 多级联调式静叶调节机构正逆运动学建模}

(1) 多级联调式静叶调节机构的正运动学模型。
式(7)表示的是第 $j$ 级静叶调节机构的正运动学 模型, 由于 $n$ 级静叶调节机构多级联动, 驱动角位 移变化量相同, 故通过 $\alpha_{j}^{0}$ 和 $\Delta \alpha_{i}$ 参数表示多级联调 式静叶调节机构的运动学模型, 其中 $\alpha_{j}^{0}$ 表示第 $j$ 级 静叶调节机构 $A B$ 杆件在驱动位置 $i=1$ 时的初始角 度; $\Delta \alpha_{i}$ 表示第 $i$ 个驱动位置相对于初始角度 $\alpha_{j}^{0}$ 的 变化量, 即可以将 $\alpha_{i, j}$ 表示为

$$
\alpha_{i, j}=\alpha_{j}^{0}+\Delta \alpha_{i}
$$

故可得到多级联调式静叶调节机构的第 $j$ 级静 叶调节机构、第 $i$ 个驱动位置的正运动学为

$$
\rho_{i, j}=g\left(\boldsymbol{x}_{j}, \alpha_{j}^{0}+\Delta \alpha_{i}\right)+\eta_{j}
$$

(2) 多级联调式静叶调节机构的逆运动学模型。

由于多级联调式静叶调节机构自由度为 1 , 给 定任意一级的输出端叶片的角位移, 通过单级静叶 调节机构的逆运动学计算可以得到输入端的角位 移。故通过式(8)和式(9)可以定义多级联调式静叶调 节机构具有解析解的逆运动学为

$$
\Delta \alpha_{i}=h\left(\boldsymbol{x}_{j}, \rho_{i, j}-\eta_{j}\right)-\alpha_{j}^{0}
$$

\section{3 正-逆运动学结合的全局设计策略}

由于多级联动调节的静叶调节机构构件数量多, 而且驱动耦合使得运动学模型非常复杂, 且与设计目 标点直接对应的机构正运动学没有解析解, 导致参数 优化设计因循环迭代求根而计算量特别大(图 5a), 使 得机构尺度参数的全局优化设计难以实现。

为避开此困境, 本文根据静叶调节机构逆运动 存在解析解的特点, 提出将正-逆运动学相结合进行 尺度全局优化设计策略, 如图 $5 \mathrm{~b}$ 所示, 把静叶调节 机构尺度优化设计问题分成两步。

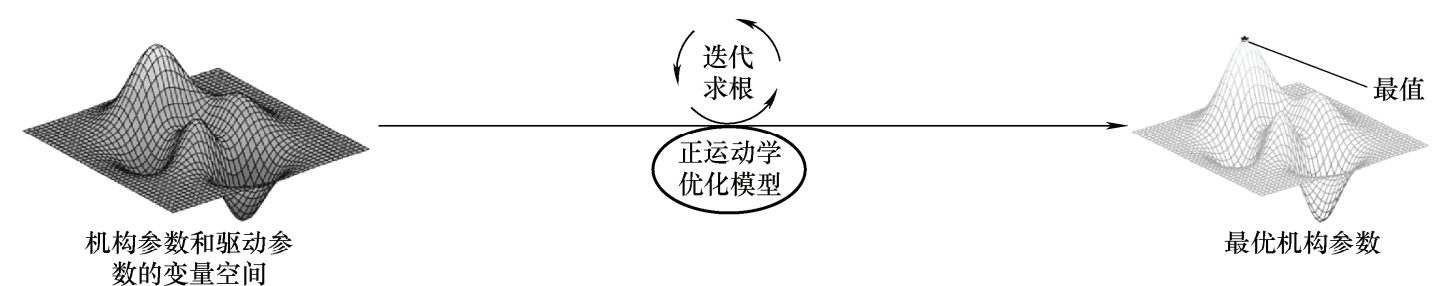

(a) 传统静叶调节机构参数全局优化设计策略

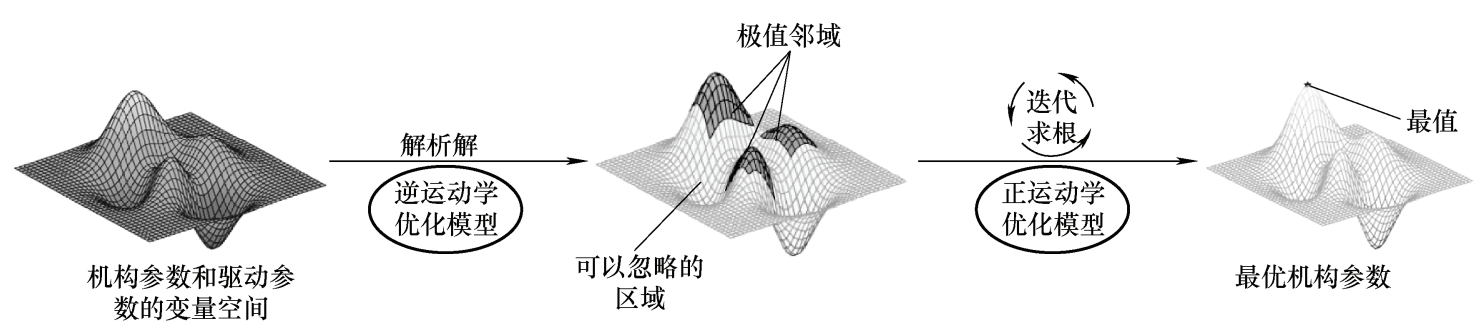

(b) 基于正-逆运动学结合的机构参数全局优化设计策略

图 5 两种设计策略的对比 
第一步, 构造基于逆运动学、具有解析解的尺 度优化模型, 采用全局优化算法, 以静叶调节规律 为输入、联动杆输出误差为评价指标, 计算出评价 指标取极值时机构参数的邻域。由于该步骤中使用 逆运动学构建优化模型, 评价指标是多级之间驱动 的同步转动误差, 故不能直接表达静叶的设计精度, 还需要构建基于正运动学、以静叶调节规律为设计 目标的优化模型。但该步骤计算求解速度快, 可以 快速锁定全局最优解所在区域, 大幅降低优化求解 的时间成本。

第二步, 构建基于正运动学数值解的高精度求 解模型, 在第一步求解得到的机构尺度参数邻域内, 采用全局优化算法, 以静叶调节规律为设计目标, 修正静叶调节机构参数。虽然本步骤采用了非解析 的正运动学模型, 但因该步骤的定义域是第一步确 定的若干极值点的邻域, 相比完整的解空间来说搜 索空间大幅缩小, 故在确保优化求解精度的同时求 解效率仍然很高。

上述正逆运动学相结合的两步式全局优化设计 方法显著缩小了正运动学优化模型求解的变量搜索 范围, 具有如下两个优势: (1)基于正-逆运动学结合 的设计方法, 降低了设计过程中的计算量, 提高了 机构参数优化设计速度和效率; (2)高效、快速的设 计方法, 使得全局优化设计策略得以实现, 提高了 全局优化设计精度, 保障了静叶调节机构这样复杂 的机构尺度设计问题得到有效解决。

\section{4 多目标优化模型建立和求解}

\section{1 构建静叶调节机构优化模型}

多目标优化问题是指一类有多个设计目标, 而且 多个目标之间存在耦合的设计问题, 这类问题一般不 可能找到一个解精确实现所有目标, 只能通过优化设 计找到多个目标均能接受的最优解。多目标优化问题 一般由决策变量、目标函数和约束条件三部分组成。 静叶调节机构设计问题是通过设计静叶调节机构参 数尺度, 使得多级静叶能够按照离散的多目标调节规 律转动, 这是一个典型的多目标优化设计问题。

根据正-逆运动学结合的全局优化策略, 构建两 种优化模型。第一种是基于机构的正运动学构建优 化模型, 通过设计联动杆角位移和机构参数, 使得 静叶的角位移按照静叶调节规律转动; 第二种是基 于机构逆运动学构建优化模型, 将静叶旋转调节的 规律作为优化模型的输入, 通过设计机构参数, 使 得多级之间联动杆转动的转角规律一致。
对于同一个静叶调节机构, 机构正、逆运动学 反映了机构的输入与输出的一一对应关系; 而基于 正、逆运动学构建的优化模型, 由于设计目标离散 等因素, 很难保证两种优化模型的误差值一一对应。 但从定性角度分析可知, 当基于逆运动学构建的优 化模型的评价指标误差减小时, 基于正运动学构件 的优化模型的评价指标也随之减小, 正、逆运动学 优化模型的极值邻域存在映射关系。

\subsection{1 基于正运动学的多目标优化模型}

（1）构建基于正运动学优化模型的决策变量。

决策变量是多目标优化问题中的自变量, 在正 运动学优化模型中, 静叶调节机构决策变量可以分 为机构参数和联动杆角位移两类。

(1) 机构参数决策变量表示为

$$
\boldsymbol{X}^{F 1}=\left[\begin{array}{ccccc}
x_{1,1} & \cdots & x_{1, j} & \cdots & x_{1, n} \\
\vdots & & \vdots & & \vdots \\
x_{k, 1} & \cdots & x_{k, j} & \cdots & x_{k, n} \\
\vdots & & \vdots & & \vdots \\
x_{p, 1} & \cdots & x_{p, j} & \cdots & x_{p, n}
\end{array}\right]
$$

式中, $\boldsymbol{X}^{F 1}$ 表示正运动学优化模型的机构参数决策 变量; $x_{k, j}$ 表示第 $j$ 级机构的第 $k$ 个机构参数; $j$ 表 示机构参数级别, 取值范围是 $j=1,2, \cdots, n ; k$ 表示 第 $k$ 个机构参数, 取值范围是 $k=1,2, \cdots, p$ 。为了方 便的描述, 用 $\boldsymbol{X}_{j}^{F 1}$ 表示第 $j$ 级所有的机构参数。

$$
\boldsymbol{X}_{j}^{F 1}=\left[\begin{array}{lllll}
x_{1, j} & \cdots & x_{k, j} & \cdots & x_{p, j}
\end{array}\right]^{\mathrm{T}}
$$

(2) 联动杆输入角位移决策变量表示为

$$
\boldsymbol{X}^{F 2}=\left[\Delta \alpha_{1}, \cdots, \Delta \alpha_{i}, \cdots, \Delta \alpha_{m}\right]^{\mathrm{T}}
$$

式中, $\boldsymbol{X}^{F 2}$ 表示正运动学优化模型联动杆输入参数 决策量。为了更加方便的表示驱动参数, $X_{i}^{F 2}$ 表示 第 $i$ 个驱动参数。

$$
X_{i}^{F 2}=\Delta \alpha_{i}
$$

（2）构建基于正运动学优化模型的目标函数。

目标函数是根据实际问题构造的关于决策变量 和设计目标之间的函数。静叶调节机构参数尺度优化 设计的正运动学优化模型, 是通过设计机构参数尺 度, 使得机构末端的构件静叶按照预定的规律转动, 故以机构驱动静叶转动规律与预定规律的误差作为 目标函数, 构造正运动学优化模型的目标函数如下

$$
\boldsymbol{Y}^{F}=\left[\begin{array}{ccccc}
\varepsilon_{1,1}^{\rho} & \cdots & \varepsilon_{1, j}^{\rho} & \cdots & \varepsilon_{1, n}^{\rho} \\
\vdots & & \vdots & & \vdots \\
\varepsilon_{i, 1}^{\rho} & \cdots & \varepsilon_{i, j}^{\rho} & \cdots & \varepsilon_{i, n}^{\rho} \\
\vdots & & \vdots & & \vdots \\
\varepsilon_{m, 1}^{\rho} & \cdots & \varepsilon_{m, j}^{\rho} & \cdots & \varepsilon_{m, n}^{\rho}
\end{array}\right]
$$


式中, $\boldsymbol{Y}^{F}$ 表示基于正运动学构建的目标函数, $\varepsilon_{i, j}^{\rho}$ 表 示第 $j$ 级静叶调节机构第 $i$ 个驱动位置的静叶角度 $\rho$ 的设计误差, 通过正运动学方程计算得到

$$
\varepsilon_{i, j}^{\rho}=g\left(\boldsymbol{X}_{j}^{F 1}, \boldsymbol{X}_{i}^{F 2}\right)+\eta_{j}-\rho_{i, j}
$$

（3）构建基于正运动学优化模型的约束条件。

约束条件是多目标优化问题关于决策变量的限 制和约束。在静叶调节机构多目标优化模型中, 机 构干涉、压气机结构尺寸等是建立约束方程的关键。 压气机结构参数不同, 约束条件也千差万别, 本文 后续在案例分析时基于实际应用讨论约束方程的建 立, 此处不再详述具体的约束方程建立的过程, 给 出约束方程的一般表达式如下

$$
\left\{\begin{array}{l}
\boldsymbol{X}_{\min }^{F 1} \leqslant \boldsymbol{X}^{F 1} \leqslant \boldsymbol{X}_{\max }^{F 1} \\
\boldsymbol{X}_{\text {min }}^{F 2} \leqslant \boldsymbol{X}^{F 2} \leqslant \boldsymbol{X}_{\text {max }}^{F 2}
\end{array}\right.
$$

\subsection{2 基于逆运动学的多目标优化模型}

（1）构建基于逆运动学优化模型的决策变量。

采用逆运动学构建优化模型, 模型的输入是静 叶调节规律, 可以作为决策变量只有机构参数。

$$
\boldsymbol{X}^{K}=\left[\begin{array}{ccccc}
x_{1,1} & \cdots & x_{1, j} & \cdots & x_{1, n} \\
\vdots & & \vdots & & \vdots \\
x_{k, 1} & \cdots & x_{k, j} & \cdots & x_{k, n} \\
\vdots & & \vdots & & \vdots \\
x_{p, 1} & \cdots & x_{p, j} & \cdots & x_{p, n}
\end{array}\right]
$$

式中, $\boldsymbol{X}^{K}$ 表示逆运动学优化模型的决策变量, 机 构参数与正运动学优化模型一致, 每个机构参数用 $x$ 表示, $x_{k, j}$ 表示第 $j$ 级机构的第 $k$ 个机构参数。为 了方便的描述, 用 $\boldsymbol{X}_{j}^{K}$ 表示第 $j$ 级所有的机构参数 如下

$$
\boldsymbol{X}_{j}^{K}=\left[\begin{array}{lllll}
x_{1, j} & \cdots & x_{k, j} & \cdots & x_{p, j}
\end{array}\right]^{\mathrm{T}}
$$

（2）构建基于逆运动学优化模型的目标函数。

静叶调节机构参数尺度优化设计的逆运动学优 化模型, 是通过设计机构参数尺度, 使得联动杆角 位移变化规律保持一致, 故以多级联动杆角位移的 误差作为目标函数。上述过程构造的逆运动学优化 模型, 设计目标是多级之间联动杆角位移误差, 不 是静叶调节规律的误差, 只是通过联动杆的误差间 接的、近似的反映了静叶调节规律的设计精度, 无 法直接表示出实际设计问题的设计精度。

构造逆运动学优化模型的目标函数如下

$$
\boldsymbol{Y}^{K}=\left[\begin{array}{ccccc}
\varepsilon_{1,1}^{\alpha} & \cdots & \varepsilon_{1, j}^{\alpha} & \cdots & \varepsilon_{1, n}^{\alpha} \\
\vdots & & \vdots & & \vdots \\
\varepsilon_{i, 1}^{\alpha} & \cdots & \varepsilon_{i, j}^{\alpha} & \cdots & \varepsilon_{i, n}^{\alpha} \\
\vdots & & \vdots & & \vdots \\
\varepsilon_{m, 1}^{\alpha} & \cdots & \varepsilon_{m, j}^{\alpha} & \cdots & \varepsilon_{m, n}^{\alpha}
\end{array}\right]
$$

式中, $\boldsymbol{Y}^{K}$ 表示基于逆运动学构建的目标函数, $\varepsilon_{i, j}^{\alpha}$ 表示第 $j$ 级静叶调节机构第 $i$ 个驱动位置的联动杆 角度 $\alpha_{i}$ 的设计误差。误差方程通过下式计算得到

$$
\varepsilon_{i, j}^{\alpha}=\left(\alpha_{j}^{0}+\Delta \alpha_{i}\right)-\frac{1}{n} \sum_{j=1}^{j=n}\left(\alpha_{j}^{0}+\Delta \alpha_{i}\right)
$$

式中, $\Delta \alpha_{i}$ 通过逆运动学式(11)计算求解。

（3）构建基于逆运动学优化模型的约束条件。

在逆运动学优化模型中, 约束方程的一般表达 式如下

$$
\boldsymbol{X}_{\min }^{K} \leqslant \boldsymbol{X}^{K} \leqslant \boldsymbol{X}_{\max }^{K}
$$

\section{2 基于正逆运动学结合的全局优化设计求解}

根据正-逆运动学优化设计求解策略, 可以提出 针对图 1 所示的静叶调节机构参数尺度全局优化设 计方法。优化模型的最值通过全局优化算法求解, 常用的全局优化算法(如遗传算法、粒子群算法和蚁 群算法等)均可用于本文的优化模型的求解, 此处以 粒子群优化算法为例介绍优化模型求解的过程, 包 含两个步骤。

第一步, 如图 6 所示, 建立静叶调节机构逆运 动学优化模型, 采用粒子群全局优化算法, 快速计 算求解出最优机构参数的邻域。由于静叶调节机构 的逆运动学是解析的, 故逆运动学优化模型计算速 度快、优化设计效率高。

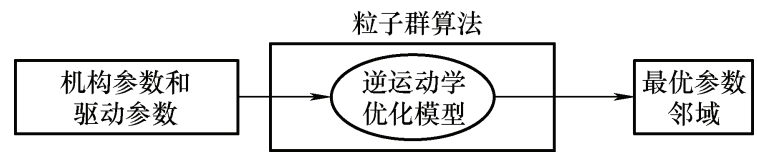

图 6 基于逆运动学优化模型的优化设计

第二步, 如图 7 所示, 建立静叶调节机构正运 动学参数优化模型, 以第一步计算的数据为初值, 采用粒子群全局优化算法, 实现机构参数的高精度 设计。由于基于正运动学的优化模型设计目标是静 叶角位移的误差, 故直接反映出静叶调节规律的设 计精度。

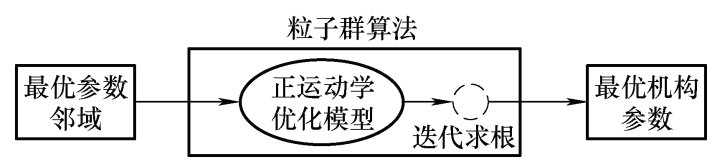

图 7 基于正运动学优化模型的优化设计

\section{5 案例分析}

\section{1 静叶调节机构尺度的全局优化设计}

以表 1 中的 5 级静叶调节规律为例, 进行静叶 调节机构的尺度设计。首先, 进行逆运动学参数优 
化, 采用粒子群优化算法, 求解全局最优机构参数, 分别得到机构参数和驱动连杆角位移的优化解; 然 后, 以第一步优化结果作为新一轮优化计算的初值, 通过正运动学优化来对静叶调节机构设计参数进行 修正, 进一步降低静叶运动误差。静叶调节机构尺 度优化设计过程可以分为五个步骤。

\section{1 .1 确定机构参数优化目标}

静叶调节机构参数优化设计的目标是静叶攻角 调节规律, 该规律一般通过然烧力学和气动力学理论 设计, 本案例设计时以表 1 的 5 级静叶攻角调节规律 为例, 设计静叶调节机构的参数, 使得静叶调节机构 的能够驱动静叶完成静叶攻角调节规律任务。

\section{1 .2 分析机构参数优化的固定参数和设计参数}

静叶调节机构中的参数主要有两类, 一类是固 定的机构参数, 这类参数在静叶调节机构参数优化 设计阶段是固定的、非设计参数, 如联动杆的安装 参数; 另一类是设计参数, 通过设计这些机构参数, 能够使得静叶攻角安装预定规律调节, 例如杆件的 长度、构件的夹角等。

\section{(1) 固定参数。}

固定参数主要是一些安装参数, 其中 $l_{H O}$ 和 $\varphi$ 参 数决定了联动杆的安装位置, $\sigma$ 和 $l_{G O}$ 决定了静叶的 转动副的位置, 故这四类参数是固定参数, 它们的 参数大小由机覀预留的安装接口确定; 又由于多级 静叶调节机构的驱动连在同一根联动杆上, 故 5 级 联调式静叶调节机构的 $l_{H O} 、 \varphi$ 和必须相同, 结合机 量预留的安装静叶调节装置的尺寸, 给出固定参数 的列表。

表 4 固定参数表

\begin{tabular}{cccccc}
\hline 机构参数 & 第 1 级 & 第 2 级 & 第 3 级 & 第 4 级 & 第 5 级 \\
\hline$l_{H O} / \mathrm{mm}$ & 500 & 500 & 500 & 500 & 500 \\
$\varphi /\left(^{\circ}\right)$ & 20 & 20 & 20 & 20 & 20 \\
$l_{G O} / \mathrm{mm}$ & 410 & 410 & 410 & 410 & 410 \\
$\sigma /\left(^{\circ}\right)$ & 90 & 90 & 90 & 90 & 90 \\
\hline
\end{tabular}

(2) 设计参数。

通过分析式(17)和式(22)的优化模型方程可知, 除去上述 4 类固定参数, 还有 8 类设计参数 (表 5), 通过设计这 8 类参数大小, 可以降低优化模型的误 差值, 使得静叶能够按照气动力学拟定的调节规律 转动。

\section{1 .3 构建机构参数约束}

通过分析参数的类型可知, 固定参数是完全约 束的参数, 在设计模型中是定值。设计参数理论上 任意取值, 但考虑到航空发动机机匣和整流罩的限
制, 设计参数通常需要根据设计目标的实际情况建 立优化模型的约束。

表 5 设计参数表

\begin{tabular}{cccccc}
\hline 机构参数 & 第 1 级 & 第 2 级 & 第 3 级 & 第 4 级 & 第 5 级 \\
\hline$l_{A H}$ & $l_{A_{1} H_{1}}$ & $l_{A_{2} H_{2}}$ & $l_{A_{3} H_{3}}$ & $l_{A_{4} H_{4}}$ & $l_{A_{5} H_{5}}$ \\
$l_{A B}$ & $l_{A_{1} B_{1}}$ & $l_{A_{2} B_{2}}$ & $l_{A_{3} B_{3}}$ & $l_{A_{4} B_{4}}$ & $l_{A_{5} B_{5}}$ \\
$l_{B C}$ & $l_{B_{1} C_{1}}$ & $l_{B_{2} C_{2}}$ & $l_{B_{3} C_{3}}$ & $l_{B_{4} C_{4}}$ & $l_{B_{5} C_{5}}$ \\
$l_{C D}$ & $l_{C_{1} D_{1}}$ & $l_{C_{2} D_{2}}$ & $l_{C_{3} D_{3}}$ & $l_{C_{4} D_{4}}$ & $l_{C_{5} D_{5}}$ \\
$\tau$ & $\tau_{1}$ & $\tau_{2}$ & $\tau_{3}$ & $\tau_{4}$ & $\tau_{5}$ \\
$l_{F G}$ & $l_{F_{1} G_{1}}$ & $l_{F_{2} G_{2}}$ & $l_{F_{3} G_{3}}$ & $l_{F_{4} G_{4}}$ & $l_{F_{5} G_{5}}$ \\
$\alpha_{j}^{0}$ & $\alpha_{1}^{0}$ & $\alpha_{2}^{0}$ & $\alpha_{3}^{0}$ & $\alpha_{4}^{0}$ & $\alpha_{5}^{0}$ \\
$\eta$ & $\eta_{1}$ & $\eta_{2}$ & $\eta_{3}$ & $\eta_{4}$ & $\eta_{5}$ \\
\hline
\end{tabular}

由于本文案例设计中设计参数有 5 级、每一级 有 8 类, 共有 40 个设计参数, 受限于篇幅长度, 此 处以第 1 级静叶调节机构的 $F_{1} G_{1}$ 连杆长度 $l_{F_{1} G_{1}}$ 参数 为例, 分析约束建立的流程和方法。

(1) 参数下限的约束。

由于连杆 $F_{1} G_{1}$ 一端连接 $\mathrm{S}$ 副、另一端连接 $\mathrm{R}$ 副, 考虑到运动副需要安装空间, 故一般取 $l_{F_{1} G_{1}} \geqslant 25 \mathrm{~mm}$ 。

(2) 参数上限的约束。

第 1 级静叶与第 2 级静叶之间的距离是连杆 $F_{1} G_{1}$ 长度最大理论值, 否则会出现第 1 级和第二级 静叶调节机构会发生干涉。根据机匣的第一级和第 二级静叶安装孔的距离, 可以确定连杆 $F_{1} G_{1}$ 上限是 $l_{F_{1} G_{1}} \leqslant 35 \mathrm{~mm}$ 。

故参数 $l_{F_{1} G_{1}}$ 约束可以表示为

$$
25 \mathrm{~mm} \leqslant l_{F_{1} G_{1}} \leqslant 35 \mathrm{~mm}
$$

采用这种分析方法可以建立其他 39 个参数约 束条件, 此处不再详述其他 39 个参数约束条件确定 的过程, 给出设计变量的约束下限和上限如表 $6 、 7$ 所示。

表 6 设计参数表下限

\begin{tabular}{cccccc}
\hline 机构参数 & 第 1 级 & 第 2 级 & 第 3 级 & 第 4 级 & 第 5 级 \\
\hline$l_{A H} / \mathrm{mm}$ & 20 & -35 & -35 & -35 & -35 \\
$l_{A B} / \mathrm{mm}$ & 35 & 35 & 35 & 35 & 35 \\
$l_{B C} / \mathrm{mm}$ & 80 & 80 & 80 & 80 & 80 \\
$l_{C D} / \mathrm{mm}$ & 440 & 430 & 420 & 430 & 440 \\
$\tau /\left(^{\circ}\right)$ & 50 & 50 & 50 & 50 & 50 \\
$l_{F G} / \mathrm{mm}$ & 25 & 25 & 25 & 25 & 25 \\
$\alpha_{j}^{0} /\left(^{\circ}\right)$ & 0 & 0 & 0 & 0 & 0 \\
$\eta /\left(^{\circ}\right)$ & 0 & 0 & 0 & 0 & 0 \\
\hline
\end{tabular}


表 7 设计参数表上限

\begin{tabular}{cccccc}
\hline 机构参数 & 第 1 级 & 第 2 级 & 第 3 级 & 第 4 级 & 第 5 级 \\
\hline$l_{A H} / \mathrm{mm}$ & 35 & -20 & -20 & -20 & -20 \\
$l_{A B} / \mathrm{mm}$ & 65 & 65 & 65 & 65 & 65 \\
$l_{B C} / \mathrm{mm}$ & 140 & 140 & 140 & 140 & 140 \\
$l_{C D} / \mathrm{mm}$ & 460 & 440 & 430 & 440 & 450 \\
$\tau /\left(^{\circ}\right)$ & 60 & 60 & 60 & 60 & 60 \\
$l_{F G} / \mathrm{mm}$ & 35 & 35 & 35 & 35 & 35 \\
$\alpha_{j}^{0} /\left(^{\circ}\right)$ & 270 & 270 & 270 & 270 & 270 \\
$\eta /\left(^{\circ}\right)$ & 360 & 360 & 360 & 360 & 360 \\
\hline
\end{tabular}

5.1 .4 机构参数优化设计求解

通过第 4 小节分析, 建立的多级联调式静叶调 节机构参数优化设计模型, 将复杂的机构参数优化 设计问题转化成数学模型, 该数学模型变量维度高, 故采用了粒子群算法求解优化模型的最优值。参数 优化设计分为逆运动学优化模型求解和正运动学优 化模型求解。

\section{(1) 逆运动学优化模型求解。}

式(22)是基于逆运动学构建的优化模型, 该优 化模型的输入参数是角度 $\boldsymbol{\rho}$, 共有 35 个, 如表 1 所 示; 固定参数有 20 个, 固定参数的取值如表 4 所示; 设计变量共有 40 个, 如表 5 所示, 设计变量的约束 上下限如表 6、7 所示。

由于式(22)具有解析解, 相比非解析的正运动 学模型, 在相同时间内能够实现更多次的优化设计, 提高了设计效率, 能够充分发挥全局优化算法的作 用。通过采用粒子群算法, 可以求解出式(22)的极 小值点。

当逆运动学优化模型取极小值时, 表明多级联 动的静叶调节机构驱动转角几乎能够保持一致。由 于逆运动学优化目标是多级调节机构的驱动同步程 度, 无法直接表示出静叶攻角调节的精度, 故还需 要再该极值点的基础上, 采用正运动学优化模型, 优化设计机构参数。

(2) 正运动学优化模型求解。

式(17)是基于正运动学构建的参数优化模型, 该优化模型的输入是驱动角度 $\boldsymbol{\alpha}$, 如表 1 中的第 2 列; 固定参数有 20 个, 取值如表 4 所示; 设计参数 有 40 个, 如表 5 所示, 设计变量的约束上下限如表 6、7 所示。

由于式(17)是非解析的, 每次优化设计都需要 大量数值迭代求解正运动学精确的运动学模型, 计 算量大、参数优化设计效率低。

在本小节(1)中已经根据逆运动学优化模型求 解出了以驱动角度 $\alpha$ 同步程度为评价指标的极值, 故在该极值邻域内, 通过正运动学优化模型, 可以
求解出以静叶攻角调节精度为评价指标的最值。

5.1.5 验证机构参数值

根据正-逆运动学结合的全局优化设计策略, 设计 得到的机构参数和驱动连杆角位移分别示于表 8、9。

表 8 基于正-逆运动学结合全局设计策略的机构参数表

\begin{tabular}{cccccc}
\hline 机构参数 & 第 1 级 & 第 2 级 & 第 3 级 & 级 & 第 5 级 \\
\hline$l_{H O} / \mathrm{mm}$ & 500.0000 & 500.0000 & 500.0000 & 500.0000 & 500.0000 \\
$\varphi /\left(^{\circ}\right)$ & 20.0000 & 20.0000 & 20.0000 & 20.0000 & 20.0000 \\
$l_{A H} / \mathrm{mm}$ & 31.7598 & -24.7759 & -24.8855 & -33.6495 & -29.7585 \\
$l_{A B} / \mathrm{mm}$ & 62.4887 & 47.7587 & 40.0089 & 40.7427 & 38.6647 \\
$l_{B C} / \mathrm{mm}$ & 121.8351 & 118.1529 & 110.4254 & 109.6364 & 105.9438 \\
$l_{C D} / \mathrm{mm}$ & 458.8870 & 437.1419 & 422.2916 & 433.4058 & 446.0362 \\
$\tau /\left(^{\circ}\right)$ & 59.0614 & 54.3983 & 57.8596 & 56.4543 & 56.4914 \\
$l_{F G} / \mathrm{mm}$ & 34.8477 & 26.0959 & 25.3693 & 34.5955 & 30.6503 \\
$l_{G O} / \mathrm{mm}$ & 410.0000 & 410.0000 & 410.0000 & 410.0000 & 410.0000 \\
$\sigma /\left(^{\circ}\right)$ & 90.0000 & 90.0000 & 90.0000 & 90.0000 & 90.0000 \\
$\alpha_{j}^{0} /\left(^{\circ}\right)$ & 30.1181 & 177.9566 & 200.6169 & 183.1421 & 178.7615 \\
$\eta /\left(^{\circ}\right)$ & 60.2738 & 243.0830 & 248.0873 & 251.3909 & 251.0865 \\
\hline & & & & &
\end{tabular}

表 9 基于正-逆运动学结合全局设计策略的驱动角位移表

\begin{tabular}{cccccccc}
\hline \multirow{2}{*}{ 驱动角度 } & \multicolumn{6}{c}{ 驱动位置序号 $i$} \\
\cline { 2 - 7 } & 1 & 2 & 3 & 4 & 5 & 6 & 7 \\
\hline$\Delta \alpha_{i} /\left(^{\circ}\right)$ & 0 & 4.0636 & 8.7821 & 13.4036 & 21.2779 & 25.4157 & 31.1258 \\
\hline
\end{tabular}

利用上述机构参数和驱动角位移规律，通过虚 拟软件仿真和数值分析, 可以得到优化设计后的静 叶调节规律，示于表 10，并绘制机构仿真曲线与设 计目标对比图像，如图 8 所示。

表 10 静叶调节规律设计值

\begin{tabular}{cccccc}
\hline $\begin{array}{c}\text { 驱动位置 } \\
\text { 序号 } i\end{array}$ & \multicolumn{5}{c}{ 静叶调节规律设计值 $/\left(^{\circ}\right)$} \\
\hline & 第 1 级 & 第 2 级 & 第 3 级 & 第 4 级 & 第 5 级 \\
\hline 1 & -0.149 & -0.120 & -0.103 & -0.062 & -0.066 \\
2 & 7.682 & 5.851 & 6.066 & 3.730 & 3.876 \\
3 & 16.771 & 12.978 & 13.245 & 8.328 & 8.655 \\
4 & 25.839 & 20.149 & 20.331 & 13.005 & 13.515 \\
5 & 42.061 & 32.853 & 32.700 & 21.315 & 22.145 \\
6 & 51.176 & 39.851 & 39.476 & 25.845 & 26.849 \\
7 & 64.770 & 50.070 & 49.401 & 32.294 & 33.547 \\
\hline
\end{tabular}

\section{2 结果分析}

根据表 1 和表 10 , 可以求得优化设计后的静叶 调节运动的偏差，示于表 11 ; 根据误差表绘制误差 曲线,示于图 9。其最大误差分别位于第 1 级的第 1 、 $4 、 5 、 7$ 位置的设计点, 第 2 级的第 $2 、 4 、 5 、 6$ 位 置的设计点, 以及第 3 级第 4、5、7 位置的设计点, 这 11 处的误差相同且均为最大误差 $\varepsilon=0.15^{\circ}$, 该设 计精度满足给定的设计要求。 


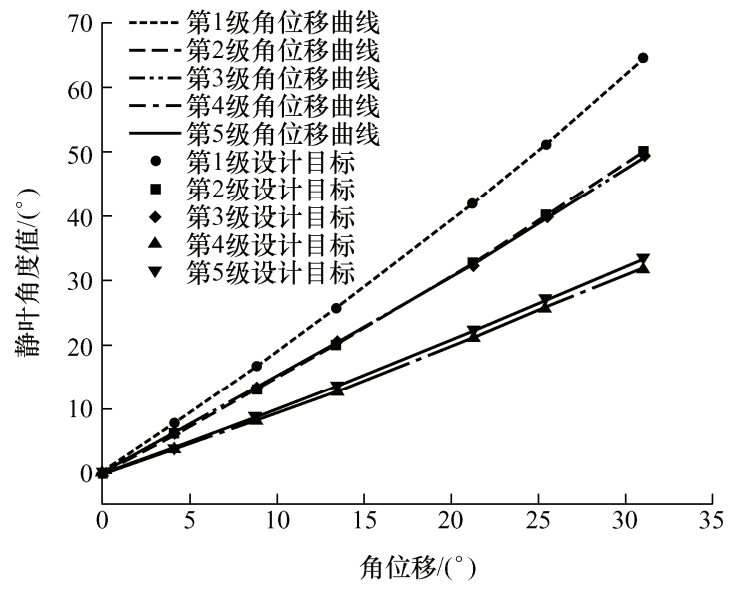

图 8 静叶调节机构输出的角位移曲线和设计目标

表 11 静叶角位移误差表

\begin{tabular}{cccccc}
\hline \multirow{2}{*}{$\begin{array}{c}\text { 驱动位置 } \\
\text { 序号 } i\end{array}$} & \multicolumn{5}{c}{ 静叶角位移误差值 $/\left(^{\circ}\right)$} \\
\hline 1 & 第 1 级 & 第 2 级 & 第 3 级 & 第 4 级 & 第 5 级 \\
\hline 1 & -0.15 & -0.12 & -0.10 & -0.06 & -0.07 \\
2 & -0.06 & -0.15 & -0.08 & -0.07 & -0.07 \\
3 & 0 & -0.02 & -0.08 & 0.01 & 0 \\
4 & 0.15 & 0.15 & -0.15 & 0.11 & 0.11 \\
5 & -0.15 & -0.15 & 0.15 & -0.11 & -0.11 \\
6 & -0.06 & -0.15 & -0.07 & -0.10 & -0.10 \\
7 & 0.15 & 0.07 & -0.15 & 0.07 & 0.07 \\
\hline
\end{tabular}

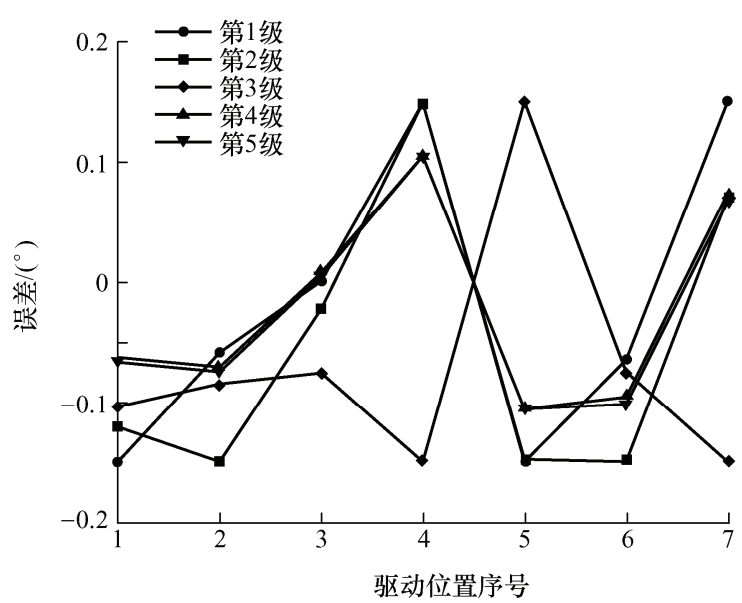

图 9 静叶角位移误差曲线图

目前的逐级分解、单级优化的局部设计方法一 般只能处理 $6 \sim 8$ 个目标点, 计算时间长, 且设计误 差在 $0.4^{\circ} \sim 0.7^{\circ}$ 范围。本文案例采用正-逆运动学结 合的全局优化方法, 处理的目标点多达 35 个, 计算 时间大幅缩短至 $20 \mathrm{~min}$ 以内, 且设计误差缩小至 $\pm 0.15^{\circ}$, 展现出本文所提的正-逆运动学结合的尺 度全局优化求解方法具有很好的实用性。

该方法为实现静叶调节机构全局参数优化提供 了新思路, 有效解决了静叶调节机构因设计参数多、 计算模型复杂等所导致的全局优化设计计算量巨 大、计算效率低的问题。
目前, 该方法已成功运用到我国某大型民用航 空发动机的高压压气机静叶调节机构的尺度优化方 案设计中, 正在进行整机原型样机的研制, 后续将 开展理论结果的试验验证工作。

\section{6 结论}

（1）根据 RSSCCSR 构型的静叶调节机构仅有 逆运动学解析解的特点, 提出正-逆运动学结合的机 构尺度全局优化设计方法。

(2) 运用所提新方法, 对 5 级联动的 R-5(SSCCSR)型静叶调节机构进行了全局优化设 计, 35 个设计目标点的误差均小于 $\pm 0.15^{\circ}$, 验证了 所提方法的有效性和实用性。

(3) 本文所提方法有效避免了目前采取的将静叶 调节机构逐级分解、单级优化的局部优化方法的不足, 有效实现了机构尺度的全局优化设计，为静叶调节机 构等复杂机构的尺度全局优化设计提供了新思路。

\section{参 考 文 献}

[1] 刘大响, 金捷. 21 世纪世界航空动力技术发展趋势与展 望[J]. 中国工程科学, 2004(9): 1-8.

LIU Daxiang, JIN Jie. The development trends and prospect of world aeropropulsion technology in the $21 \mathrm{st}$ century[J]. Engineering Science, 2004(9): 1-8.

[2] 郭令. 变循环发动机变几何部件机构学设计方法与新 机型研究[D]. 上海: 上海交通大学, 2014.

GUO Ling. On the mechanism design method for variable geometry component in variable cycle engine[D]. Shanghai: Shanghai Jiao Tong university, 2014.

[3] MILLS J P, RUDDY J M. Independently-controlled gas turbine inlet guide vanes and variable stator vanes: U.S. Patent 9,068,470[P]. 2015-06-30.

[4] 孙凯, 林清松, 张屹尚. 基于 ADAMS 与 ISIGHT 的压 气机 VSV 调节机构运动学优化设计 $[\mathrm{C}] / /$ 探索创新交 流(第 7 集). 第七届中国航空学会青年科技论坛, 中山, 2016: 364-368.

SUN Kai, LIN Qingsong, ZHANG Yishang. Kinematic optimization of compressor VSV system base on ADAMS and ISIGHT[C]. The Seventh China Aviation Society Youth Science and Technology Forum. Zhongshan, 2016: 364-368.

[5] 梁爽, 印雪梅, 王华. 基于 ADAMS 的静叶联动调节机 构参数化设计 [J]. 航空发动机, 2016, 42(1): 65-69. LIANG Shuang, YIN Xuemei, WANG Hua. Parametric design of stator blade jointly adjusting mechanism based 
on ADAMS[J]. Aeroengine, 2016, 42(1): 65-69.

[6] 杨伟, 徐伟. ADAMS 参数化分析在高压压气机调节机 构设计中的初步应用 $[\mathrm{J}]$. 燃气浴轮试验与研究, 2012, 25(4): 20-24.

YANG Wei, XU Wei, Preliminary application of parameterized analysis based on ADAMS in VSV's adjusting mechanism design of high pressure compressor[J]. Gas Turbine Experiment and Research，2012，25(4): 20-24.

[7] 贺飞, 陈国智, 温泉, 等. 浴轴发动机叶片调节机构设 计及应用 $[\mathrm{J}]$. 航空动力学报, 2007(2): 332-336.

HE Fei, CHEN Guozhi, WEN Quan, et al. Design of the control mechanism for the multistage axial compressor variable vane[J]. Journal of Aerospace Power, 2007(2): 332-336.

[8] WIRKOWSKI P. Influence of the incorrect settings of axial compressor inlet variable stator vanes on gas turbine engine work parameters[J]. Journal of KONES, 2012, 19: 483-489.

[9] 陈禁章. 中国航空发动机高压压气机发展的几个问题 [J]. 航空发动机, 2006(2): 5-11， 37.

CHEN Maozhang. Some issues in the research and development of aeroengine HP compressor in China[J]. Aeroengine, 2006(2): 5-11, 37.

[10] 程荣辉. 轴流压气机设计技术的发展 [J]. 燃气浴轮试 验与研究, 2004(2): 1-8.

CHENG Ronghui. Development of design technology for axial compressor[J]. Gas Turbine Experiment and Research, 2004(2): $1-8$.
[11] 廖吉香, 姜斌, 吕从鹏, 等. 多级轴流压气机多排可转 导/静叶联合调节规律研究[J]. 推进技术, 2017, 38(2): 334-340.

LIAO Jixiang, JIANG Bin, LÜ Congpeng, et al. Numerical optimization of combined adjustment of multirow variable inlet guide vane and stators in a multistage axial-flow compressor[J]. Journal of Propulsion Technology, 2017, 38(2): 334-340.

[12] WANG Z, FAN K, MA W, et al. Research on optimal matching method of variable stator vanes for multi-stage compressor based on genetic algorithm[C]// ASME Turbo Expo 2018: Turbomachinery Technical Conference and Exposition. American Society of Mechanical Engineers, 2018: V003T23A005-V003T23A005.

[13] WANG Z, LI J, FAN K, et al. The off-design performance simulation of marine gas turbine based on optimum scheduling of variable stator vanes[J]. Mathematical Problems in Engineering, 2017, 1: 1-11.

[14] 王华强, 王英锋, 常炜, 等. 静叶调节对多级轴流压缩 机性能影响的分析[J]. 风机技术，2017，59(3)：8-13. WANG Huaqiang, WANG Yingfeng, CHANG Wei, et al. The effect of variable static blade adjustable on multistage axial compressor performance[J]. Chinese Journal of Turbomachinery, 2017, 59(3): 8-13.

作者简介: 唐佑远, 男, 1990 年出生。主要研究方向为机构学。

E-mail: youyuantang@foxmail.com

郭为忠(通信作者), 男, 1970 年出生, 博士, 教授, 博士研究生导师。 主要研究方向为并联机构学、重大装备创新设计等。

E-mail: wzguo@sjtu.edu.cn 